Review began 01/29/2022 Review ended 02/22/2022 Published 03/06/2022

๑) Copyright 2022

Peter et al. This is an open access article distributed under the terms of the Creative Commons Attribution License CC-BY 4.0. which permits unrestricted use, distribution, and reproduction in any medium, provided the original author and source are credited.

\section{Risk Factors Associated With COVID-19 Symptoms and Potential Vertical Transmission During Pregnancy: A Retrospective Cohort Study}

Bibita Peter ${ }^{1}$, NIcholas Ree ${ }^{2}$, Karen Ferrer ${ }^{2}$, Laila Younes ${ }^{3}$, Barbara Lepe ${ }^{4}$, Khilfeh Manhal ${ }^{1}$, Janardhan Mydam $^{1}$

1. Neonatology, John H. Stroger, Jr. Hospital of Cook County, Chicago, USA 2. Pathology, John H. Stroger, Jr. Hospital of Cook County, Chicago, USA 3. Family Medicine, AMITA Health Saints Mary and Elizabeth Hospital, Chicago, USA 4. Maternal Child Services, AMITA Health Saints Mary and Elizabeth Hospital, Chicago, USA

Corresponding author: Janardhan Mydam, mydamj@gmail.com

\section{Abstract}

\section{Objective}

The COVID-19 pandemic is of special concern for pregnant women. A growing body of evidence suggests the virus can have a deleterious impact upon outcomes related to birth and newborn health. There is a paucity of published research demonstrating the factors that influence disease severity among those who are pregnant, while a growing body of evidence demonstrates that vertical transmission occurs. Our study investigated the impact of maternal characteristics upon COVID-19 outcomes, as well as whether disease severity impacted pregnancy outcomes.

\section{Methods}

We conducted a retrospective cohort study of pregnant women with COVID-19 who were admitted to two public hospitals in our state between April-August, 2020. Pregnancy outcomes and clinical, laboratory, and placental data were collected.

\section{Results}

Thirty-four pregnant women tested positive for SARS-CoV-2. Among them, 55\% (19/34) were symptomatic. Of those who were symptomatic, 68\% (13/19) presented with fever and cough. Those with symptoms had a statistically significant higher pregestational mean body mass index (BMI) compared with asymptomatic women (35.7 \pm 7.9 vs $26.7 \pm 6.9, P=0.004)$. Screening of biochemical records demonstrated that symptomatic women had lower potassium levels compared with those who were asymptomatic (median: $3.70 \mathrm{mEq} / \mathrm{L}$ vs $4.30 \mathrm{mEq} / \mathrm{L}, P=0.009)$. The lowest potassium level $(3.0 \mathrm{mEq} / \mathrm{L})$ and one of the highest BMIs $\left(42.4 \mathrm{~kg} / \mathrm{m}^{2}\right)$ was observed in the only case of postpartum mortality among the symptomatic women. We did not observe any influence of maternal COVID-19 severity on placental histopathology/infant health or evidence of vertical transmission.

\section{Conclusion}

High pregestational BMI and lower potassium levels were associated with the presence of COVID-19 symptoms among pregnant women.

Categories: Obstetrics/Gynecology, Pediatrics, Infectious Disease

Keywords: sars-cov-2, pregnancy, hypokalemic, potassium, overweight, obesity, bmi, covid-19

\section{Introduction}

As the global impact of coronavirus disease 2019 (COVID-19) has grown, so has our understanding of the plethora of presenting symptoms. Known symptoms now include anosmia, myalgia, and gastrointestinal symptoms, including diarrhea, vomiting, nausea, and abdominal pain, in addition to respiratory symptoms of fever, dry cough, and dyspnea [1-3]. It is also established that older age, underlying medical conditions (immunosuppression, hypertension, and diabetes), and race/ethnicity (Black, Hispanic and Asian) increase the risk of severe disease [4-6]. However, the impact of COVID-19 and associated risk factors on pregnancy and newborn health remains less certain.

A number of studies, including a large systematic review, suggest that the overall risk of vertical transmission and clinical symptoms in newborns of women with COVID-19 is small [1, 7, 8], although risks of neonatal complications are higher compared to infants of non-infected mothers [9]. However, evidence of the considerable effects of COVID-19 on maternal morbidity and mortality continues to accumulate $[10,11]$. A multinational study showed an increased risk of pre-eclampsia, severe infections, intensive care unit 
admission, preterm birth, and maternal mortality in pregnant women with COVID-19, relative to their uninfected counterparts [9]. With the recent implementation of aggressive COVID-19 testing, several reports have emerged associating factors such as pregestational body mass index (BMI), lymphocyte count, heart rate, and respiratory rate with the development of disease symptoms in SARS CoV-2-infected pregnant women [12-16]. Another study demonstrated an increased risk of preterm delivery in symptomatic women, suggesting a need to identify high-risk pregnant women [10]. One of the potential reasons for the increased risk could be damage to placental tissue [16].

Our study sought to investigate the impact of maternal demographic, clinical and biochemical characteristics on disease severity in pregnant women infected with SARS-Cov-2. The ability to identify factors that place pregnant women at particular risk of developing COVID-19 symptoms may help healthcare providers determine which women need to be closely monitored for a potential rapid progression of the illness. We further investigated the influence of disease severity on pregnancy outcomes, vertical transmission of infection, histopathological evaluation of the placenta, and outcomes of the newborn.

This article appeared as a preprint on Research Square on October 1, 2021. It was also presented as an electronic poster at the 2021 virtual meeting of the Pediatric Academic Societies (PAS 2021).

\section{Materials And Methods Study design and study population}

During the peak of the first wave of COVID-19 infections in the US, testing for COVID-19 became universal for all pregnancy admissions from April 2020 in Illinois, USA. We conducted a retrospective cohort study between April 1 and August 15, 2020, that included all pregnant women who were admitted at any gestational age, at any stage of labor, and had laboratory-confirmed COVID-19. Admissions were from two Chicago maternity hospitals - John H. Stroger, Jr. Hospital of Cook County and AMITA Health Saints Mary and Elizabeth Medical Center. Our study was possible because of the April 2020 implementation of universal COVID-19 testing for all pregnant women admitted to hospitals in Illinois. We followed the World Health Organization (WHO) guidelines for diagnosis, which define positive real-time reverse transcriptasepolymerase chain reaction (RT-PCR) assay of nasal or pharyngeal swabs as laboratory-confirmed SARS-CoV2 [17]. We excluded patients lacking the data on COVID-19 related symptoms (fever, cough, myalgia, anosmia, congestion, headache, chills, dyspnea, nausea, vomiting, or malaise) that would allow us to classify them as symptomatic or asymptomatic. We also excluded one woman with missing data on pregnancy-related complications, such as pregnancy-induced hypertension, chronic hypertension, pregnancy-related diabetes, or chronic diabetes (she did not have prenatal care). After the selection of the study population, we further included their newborns in the study.

\section{Ethical approval and data collection}

The study's protocol was expeditiously approved by the institutional research ethics committees associated with John H. Stroger, Jr. Hospital of Cook County (approval number: 20-098) and AMITA Health Saints Mary and Elizabeth Medical Center (approval number: 2021-0193-02). The requirement of informed consent was waived due to the retrospective study design.

The following demographic and baseline maternal data were collected: age, race and ethnicity, BMI, lifestyle habits (substance abuse, alcohol consumption, and smoking), comorbidities including pre-gestational obesity, hypertension, gestational diabetes, and whether the patient received antepartum therapy including hydroxychloroquine treatment. All participants underwent clinical evaluation of presenting signs and symptoms, detailed laboratory assessment of blood and urine samples, and radiologic chest assessment if needed.

Maternal blood sample assessment included hemoglobin, blood cell counts, inflammatory markers [e.g., Creactive protein (CRP)], serum concentration of electrolytes (sodium, potassium, calcium, and chloride), liver function (alanine aminotransferase [ALT], aspartate aminotransferase [AST]), and renal function (blood urea nitrogen $[\mathrm{BUN}]$ and creatinine). Data on pregnancy outcomes (including mode of delivery, gestational age (GA), and preeclampsia), and neonatal outcomes [including symptoms, Appearance, Pulse, Grimace, Activity, and Respiration (APGAR) scores, and birth weight] were recorded. Preterm birth or premature birth was defined as one occurring at < 37 weeks. We used specific cut-off values to define blood dyscrasias for pregnant women according to their trimester: leukopenia as white cell count $<5.9 \times 109 / \mathrm{L}$, neutropenia as neutrophil count < $3.9 \times 109 / \mathrm{L}$, and lymphopenia as lymphocyte count < $1.0 \times 109 / \mathrm{L}$ [18] for the third trimester. We also performed the gross and histopathological evaluation of placentas according to the Amsterdam consensus statement guidelines [19].

We further performed laboratory assessment of blood samples taken from the newborns and followed up both mother and infant until six weeks after delivery. We defined the infant's specific blood dyscrasias according to their age of life: leukopenia as white cell count $<13.0 \times 109 / \mathrm{L}$ for term infants at one to 12 hours of life and < $9.0 \times 109 / \mathrm{L}$ for preterm infants at birth; neutropenia as neutrophil count < $6.0 \times 109 / \mathrm{L}$ for term infants at one to 12 hours of life and < $6.0 \times 109 / \mathrm{L}$ for preterm infants at birth; and lymphopenia as 
lymphocyte count $<2.0 \times 109 / \mathrm{L}$ for both term infants at one to 12 hours of life and preterm infants at birth [20]. Evidence of vertical transmission was further evaluated for the presence of SARS-CoV-2 according to CDC guidelines [21]. The guidelines require two PCR tests, the first at 24 hours and the second at 48 hours after birth, along with clinical monitoring for signs and symptoms of COVID-19. Our protocol for prevention of perinatal infection initially included separation of newborns from mothers (who were SARS-CoV-2 positive) after delivery, but later CDC guidelines left the decision to separate up to the mothers. In our participating hospitals, mothers who chose to care for their babies were instructed to practice strict handwashing and masking and to maintain social distancing ( 6 feet) when not caring for the baby, for either 14 days or four days following resolution of symptoms. As, and when, appropriate we evaluated infants for immunoglobulin [Ig] G and IgM levels.

All the data collected was curated using a customized data collection form, and two study investigators (JM and BP) independently reviewed the data collection forms for any errors. The data was locked and secured appropriately according to rules and principles laid down in the Health Insurance Portability and Accountability Act of 1996 (HIPAA). The data collected from both sites were synchronized and any inaccuracies were verified with the concerned representative of the specific center.

\section{Study outcome}

The patients were further classified into two groups: symptomatic and asymptomatic, according to the existence of any of the following known signs and symptoms of COVID-19 infection: fever, cough, myalgia, anosmia, congestion, headache, chills, desaturation, dyspnea, nausea, vomiting, or malaise. The symptomatic group included women who displayed symptoms at any time before or after delivery.

\section{Statistical analyses}

Statistical analyses were conducted using STATA 16 (StataCorp. 2019. Stata Statistical Software: Release 16. College Station, TX: StataCorp LLC) and SAS 9.4 (SAS Institute, Inc., Cary, NC). Continuous variables were expressed as mean and standard deviation (SD) for normally distributed data, median and range for nonnormally distributed data, and categorical variables as frequency and percentage. The Shapiro-Wilk, Kolmogorov-Smirnov, Cramer-von Mise, and Anderson-Darling tests were used to check the normality of the data. The continuous variables were compared between symptomatic and asymptomatic individuals using a t-test or Mann-Whitney-U test and categorical variables were compared using the Pearson chi-square test or Fisher's-exact test, as appropriate. We calculated the unadjusted odds ratio (OR) using the simple logistic regression model. A p-value of $<0.05$ was considered statistically significant.

\section{Results}

A total of 37 pregnant women with COVID-19 were admitted to the study centers during the study period. One patient was excluded for lack of information on the existence of symptoms, while two were excluded due to lack of data related to maternal and perinatal outcomes. In total, 34 women were included in the study, and 19 were symptomatic (55\%) for COVID-19 (Figure 1). 


\section{Cureus}

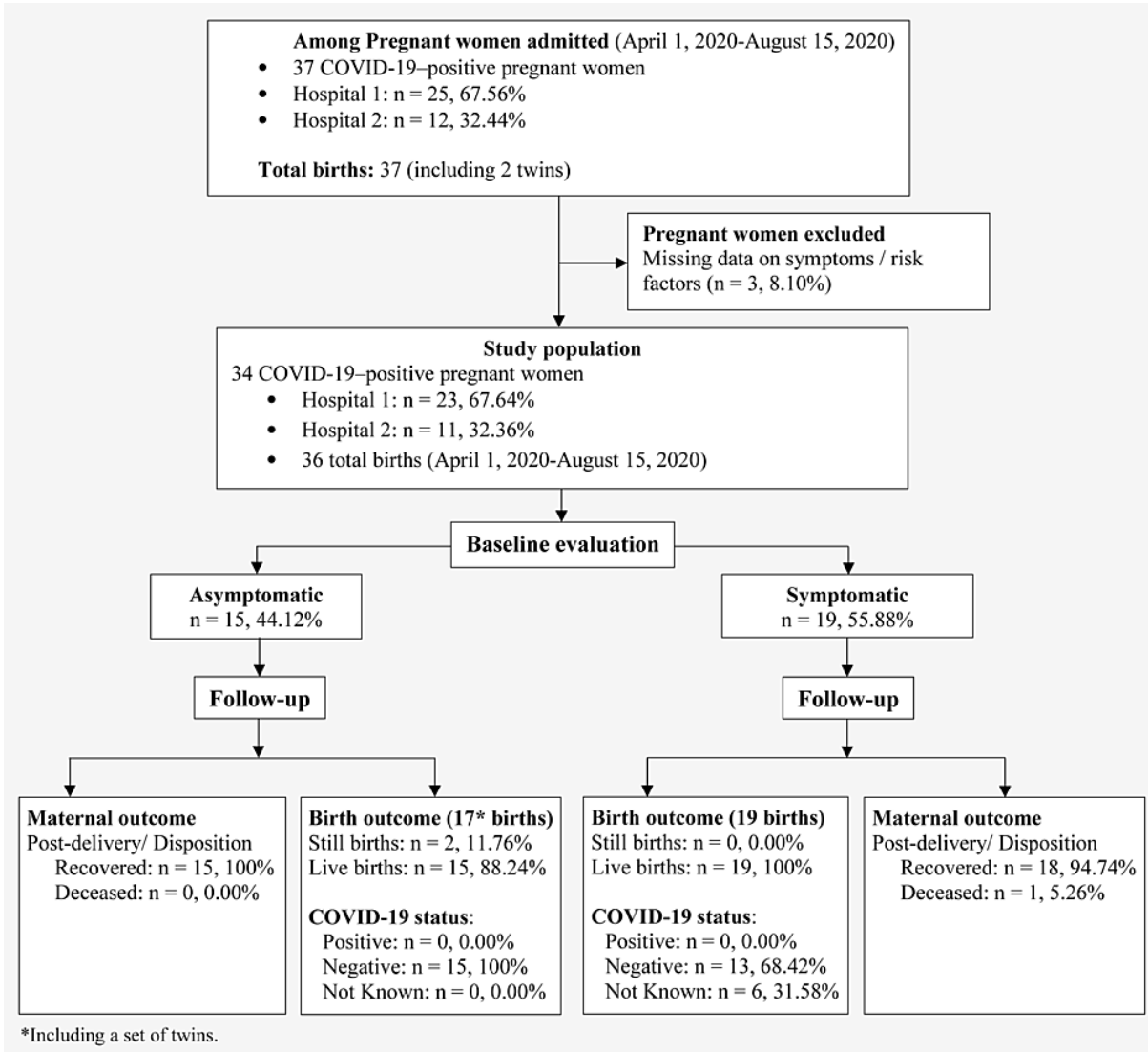

FIGURE 1: Flowchart of the study population selection and maternal and infant outcomes. Hospital 1 is John H. Stroger, Jr. Hospital of Cook County; Hospital 2 is AMITA Health Saints Mary and Elizabeth Medical Center.

\section{Baseline characteristics of pregnant women}

The baseline characteristics of the study population are shown in Table 1. Among the 19 symptomatic patients, fever $(n=13 ; 68 \%)$, cough $(n=8 ; 42 \%)$, and myalgia $(n=5 ; 26 \%)$ were the most commonly observed symptoms (Figure 2). Fever, cough, and myalgia were the most frequently observed combination of coexisting symptoms, seen in three patients (15.79\%; data not shown). The average age of women in our study population was 26 . The largest proportion was of Hispanic ancestry (44\%), followed by non-Hispanic Black (38\%). The distribution of age and race and ethnicity did not differ significantly between symptomatic and asymptomatic patients.

\begin{tabular}{|c|c|c|c|c|c|}
\hline \multirow{2}{*}{ Characteristics } & \multicolumn{2}{|c|}{ Asymptomatic (N=15) } & \multicolumn{2}{|c|}{ Symptomatic (N=19) } & \multirow{2}{*}{$\mathbf{P}$} \\
\hline & $\mathbf{n}$ & Mean (SD)/ Median (IQR)/ \% & $\mathbf{n}$ & Mean (SD)/ Median (IQR)/ \% & \\
\hline Age at diagnosis ${ }^{a}$ & 15 & $24.93(5.09)$ & 19 & $27.32(5.96)$ & 0.227 \\
\hline \multicolumn{6}{|l|}{ Gravidity ${ }^{c}$} \\
\hline$>1$ & 9 & 60.00 & 15 & 78.95 & 0.276 \\
\hline \multicolumn{6}{|l|}{ Parity $^{c}$} \\
\hline$>1$ & 5 & 33.33 & 8 & 42.11 & 0.601 \\
\hline GA at diagnosis (weeks) ${ }^{b}$ & 15 & $39.00(38.40-39.10)$ & 18 & $35.90(32.60-39.10)$ & 0.173 \\
\hline GA at delivery (weeks) ${ }^{b}$ & 14 & $39.05(38.50-40.30)$ & 19 & $39.10(37.50-40.00)$ & 1.000 \\
\hline
\end{tabular}




\section{Cureus}

\begin{tabular}{|c|c|c|c|c|c|}
\hline \multirow{2}{*}{$\begin{array}{l}\text { Diagnosis to delivery interval (weeks) } \\
\text { beharacteristics } \\
\text { Ethnicity } \\
\text { Hispanic }\end{array}$} & \multicolumn{2}{|c|}{$\begin{array}{l}14 \quad 0.05(0-0.80) \\
\text { Assymptomatic }(\mathrm{N}=15)\end{array}$} & \multicolumn{2}{|c|}{$\begin{array}{l}19 \\
\text { Symptomatic }(\mathrm{N}=190)\end{array}$} & \multirow{2}{*}{$\begin{array}{c}0.035 \\
\mathbf{P}\end{array}$} \\
\hline & $7^{\mathbf{n}}$ & $\begin{array}{l}\text { Mean (SD)/ Median (IQR)/ \% } \\
46.67\end{array}$ & $8^{\mathbf{n}}$ & $\begin{array}{l}\text { Mean (SD)/ Median (IQR)/ \% } \\
42.11\end{array}$ & \\
\hline Non-Hispanic black & 6 & 40.00 & 7 & 36.84 & \multirow{3}{*}{1.000} \\
\hline Non-Hispanic white & 0 & 0.00 & 1 & 5.26 & \\
\hline Other & 2 & 13.33 & 3 & 15.79 & \\
\hline \multicolumn{6}{|l|}{ Complications during pregnancy } \\
\hline Pre-pregnancy BMI (kg/m2) ${ }^{a}$ & 14 & $26.79(6.92)$ & 14 & $35.71(7.91)$ & 0.004 \\
\hline Obesity $(\mathrm{BMI}>30)^{\mathrm{C}}$ & 0 & 0.00 & 5 & 26.32 & 0.032 \\
\hline GBS positive $^{C}$ & 4 & 26.67 & 7 & 36.84 & 0.715 \\
\hline HIV positive ${ }^{C}$ & 0 & 0.0 & 2 & 10.53 & 0.492 \\
\hline Preeclampsia ${ }^{\mathrm{C}}$ & 2 & 13.33 & 2 & 10.53 & 1.000 \\
\hline $\mathrm{HTN}^{\mathrm{C}}$ & 1 & 6.67 & 5 & 26.32 & 0.196 \\
\hline Cholestasis $^{c}$ & 0 & 0.00 & 2 & 10.53 & 0.492 \\
\hline Chorioamnionitis $^{c}$ & 0 & 0.00 & 3 & 15.79 & 0.244 \\
\hline Anemia $^{c}$ & 0 & 0.00 & 1 & 5.26 & 1.000 \\
\hline Gestational diabetes $^{c}$ & 0 & 0.00 & 2 & 10.53 & 0.492 \\
\hline Substance abuse ${ }^{C}$ & 1 & 6.67 & 2 & 10.53 & 1.000 \\
\hline Depression $^{\mathrm{C}}$ & 0 & 0.00 & 1 & 5.26 & 1.000 \\
\hline History of syphilis ${ }^{c}$ & 1 & 6.67 & 0 & 0.00 & 0.441 \\
\hline History of HSV ${ }^{\mathrm{C}}$ & 0 & 0.00 & 1 & 5.26 & 1.000 \\
\hline Oligohydramnios ${ }^{C}$ & 0 & 0.00 & 1 & 5.26 & 1.000 \\
\hline \multicolumn{6}{|l|}{ Relevant Antepartum therapy ${ }^{c}$} \\
\hline Flu vaccination in pregnancy & 3 & 20.00 & 9 & 47.37 & 0.097 \\
\hline History of Malaria medication & 1 & 6.25 & 0 & 0.00 & NA \\
\hline Antiviral & 0 & 0.00 & 0 & 0.00 & NA \\
\hline Hydroxychloroquine & 0 & 0.00 & 1 & 5.26 & NA \\
\hline Betamethasone & 2 & 13.33 & 0 & 0.00 & 0.187 \\
\hline \multicolumn{6}{|l|}{ Labor and delivery } \\
\hline \multicolumn{6}{|l|}{ Mode of delivery ${ }^{c}$} \\
\hline Vaginal delivery & 11 & 73.33 & 14 & 73.68 & \multirow{2}{*}{1.000} \\
\hline Cesarean delivery & 4 & 26.67 & 5 & 26.32 & \\
\hline \multicolumn{6}{|l|}{ Multiplicity of birth ${ }^{c}$} \\
\hline Twins (n, \%) & 2 & 13.33 & 0 & 0.00 & 0.187 \\
\hline \multicolumn{6}{|l|}{ Disposition/ Recovery ${ }^{c}$} \\
\hline Postpartum death, n (\%) & 0 & 0.00 & 1 & 5.26 & 1.000 \\
\hline
\end{tabular}




\section{Cureus}

TABLE 1: Clinical and demographic characteristics of pregnant women with COVID-19 by

\section{symptom status}

BMI: body mass index; GA: gestational age; GBS: Group B Streptococcus; HIV: human immunodeficiency virus; HSV: herpes simplex virus; HTN: hypertension; NA: not available; SD: standard deviation, IQR: Interquartile range.

The Shapiro-Wilk, Kolmogorov-Smirnov, Cramer-von Mises, and Anderson-Darling tests were used to check the normality of the data.

${ }^{\mathrm{a}}$ Mean (SD), ${ }^{\mathrm{b}}$ Median (IQR), ${ }^{\mathrm{c}}$ Percentage (\%)

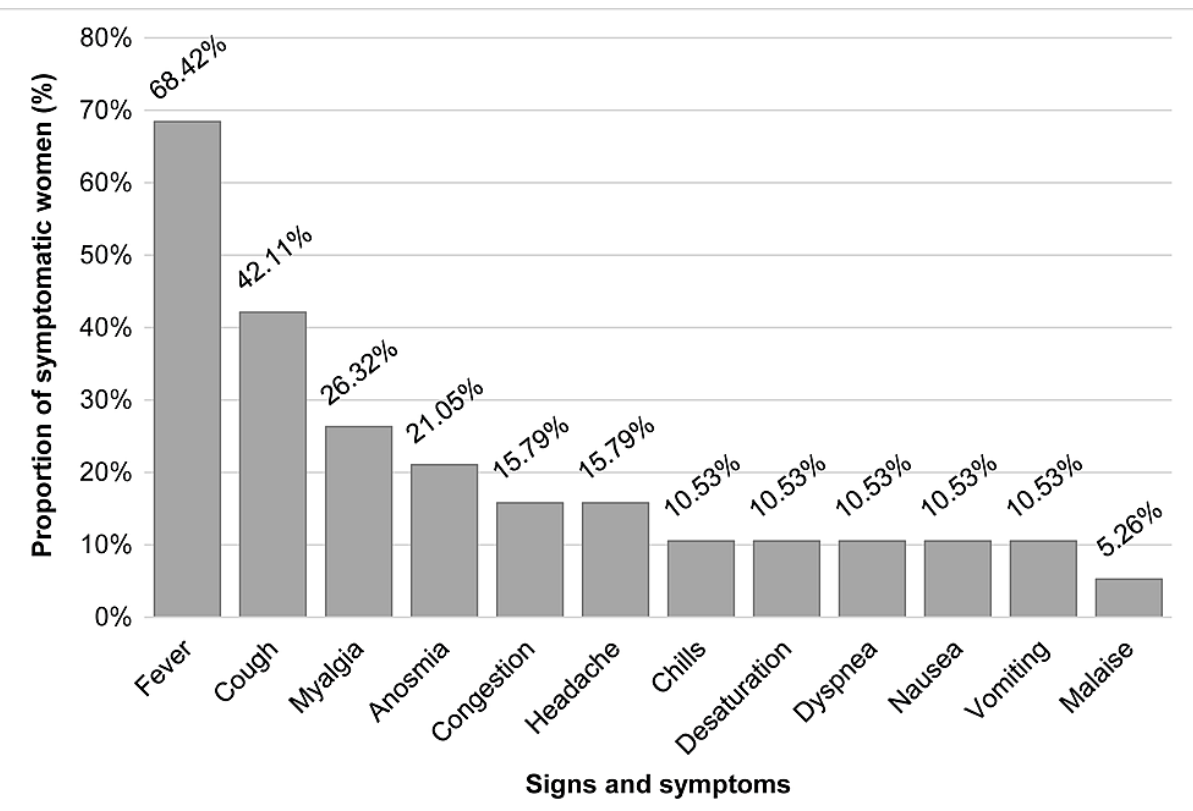

FIGURE 2: Distribution of symptoms, secondary to COVID-19 infection, among symptomatic pregnant women.

Obesity ( $n=5 ; 26.32 \%)$, gestational diabetes $(n=2 ; 10.53 \%)$, and hypertension $(n=5 ; 26.32 \%)$ were the most commonly occurring comorbid conditions. All three were overrepresented in symptomatic patients. We also observed that the symptomatic women had significantly higher pre-gestational BMI compared with asymptomatic women ( 35.71 vs $26.79, \mathrm{P}=0.004$; Figure $3 \mathrm{~A}$ ). Our posthoc power analysis for BMI with our sample size ( $\mathrm{n}=34)$, mean difference of 8.92, standard deviations of 6.92 (group 1) and 7.91 (group 2), and an alpha error $<0.05$ showed $92 \%$ as the power of the study. 


\section{Cureus}

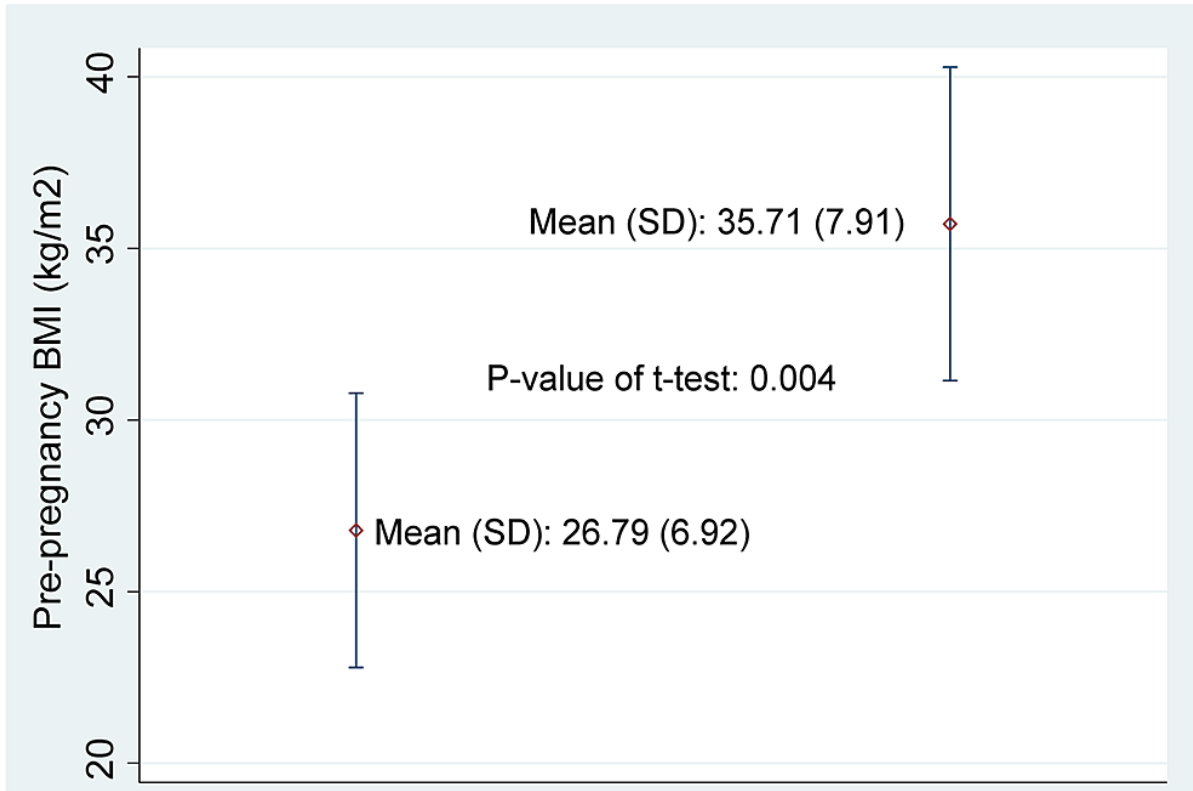

A

Asymptomatic

Group

Symptomatic

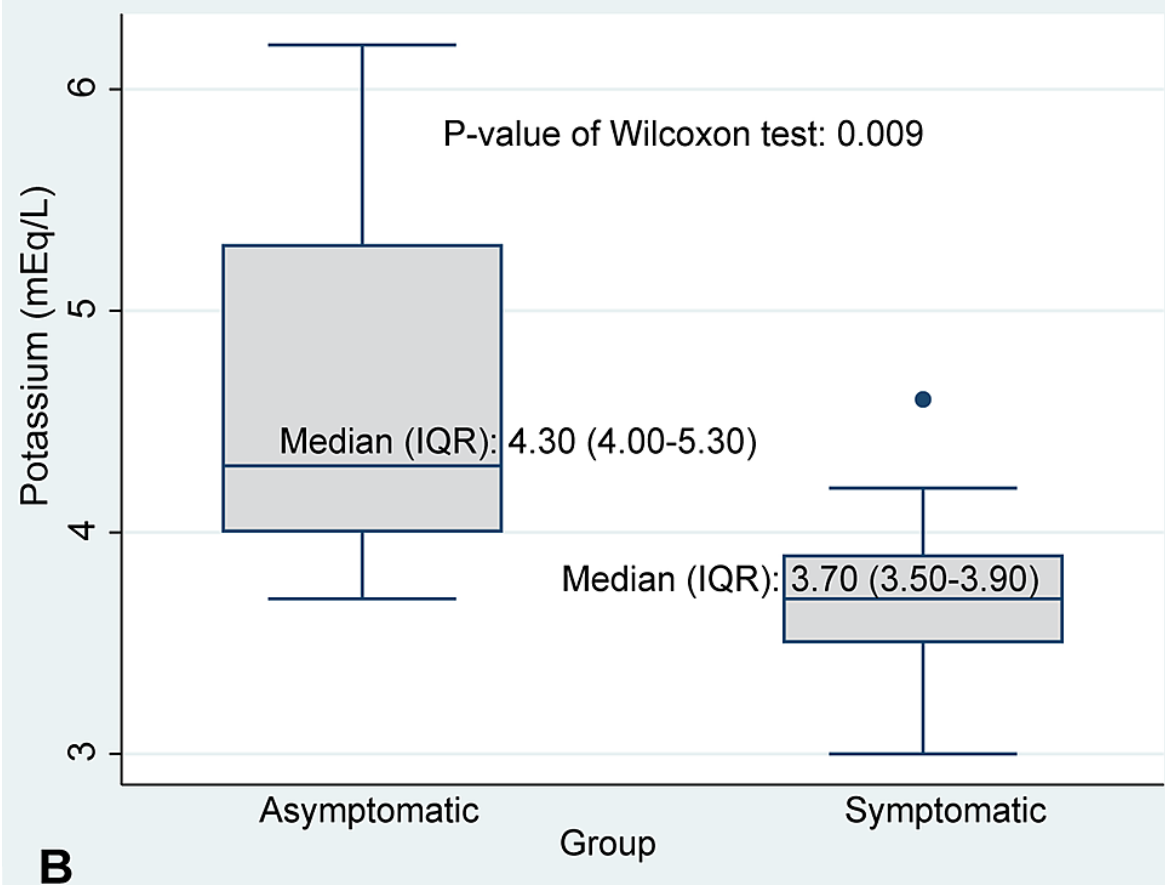

FIGURE 3: Distribution of pregestational BMI and potassium levels among pregnant women with COVID-19.

A. Distribution of pregestational BMI by symptom status; B. Distribution of potassium levels by symptom status.

Two of our symptomatic patients were also positive for HIV. Another three symptomatic women were diagnosed with chorioamnionitis. A comparatively large proportion of symptomatic women in our cohort had received the flu vaccination in the recent past (47.37\% of symptomatic women vs $20.00 \%$ of asymptomatic women). We further observed that every third patient in our enrolled population was a Group B streptococcus (GBS) carrier; however, we failed to detect any influence of GBS status on the absence or presence of symptoms. Our cohort also had two women with a history of substance abuse, one symptomatic and one asymptomatic. We also detected syphilis in one of the asymptomatic patients.

We failed to detect any significant difference in the method or mode of delivery in symptomatic vs 


\section{Cureus}

asymptomatic women $(\mathrm{P}=0.493)$. Of the four cesarean deliveries performed in asymptomatic women, two were for twins in a breech presentation and two were indicated by non-reassuring fetal status.

\section{Laboratory characteristics of pregnant women}

Initial evaluation of laboratory characteristics of the study population is shown in Table 2. Hematological analyses suggested significantly elevated basophil counts in symptomatic, compared with asymptomatic, women $(\mathrm{P}=0.035)$. However, we failed to observe any significant difference in blood dyscrasias, including leukopenia, neutropenia, and lymphopenia. No differences in kidney or liver function were observed. Symptomatic women had significantly lower potassium levels compared to asymptomatic women (median: $3.70 \mathrm{mEq} / \mathrm{L}$ [IQR: 3.50-3.90] vs. median $4.30 \mathrm{mEq} / \mathrm{L}$ [IQR: 4.00-5.30]; $\mathrm{P}=0.009$; Figure 3B). The effect of lower potassium levels on disease severity was further observed to have a more profound effect in the presence of high BMI. We observed both low potassium levels (3.0 mEq/L, the lowest in the study) and high BMI (42.4 $\mathrm{kg} / \mathrm{m} 2$, one of the highest in the cohort), in the only case of postpartum mortality among the symptomatic women in our study population. Three women, all of them symptomatic, showed evidence of secondary infection, with positive cell $(\mathrm{n}=1)$ and urine $(\mathrm{n}=2)$ cultures.

\begin{tabular}{|c|c|c|c|c|c|c|}
\hline \multirow{2}{*}{ Characteristics } & \multirow{2}{*}{ Normal range } & \multicolumn{2}{|c|}{ Asymptomatic $(\mathrm{N}=15)$} & \multicolumn{2}{|c|}{ Symptomatic $(\mathrm{N}=19)$} & \multirow{2}{*}{$\mathbf{P}$} \\
\hline & & $\mathbf{n}$ & Median (IQR)/ \% & $\mathbf{n}$ & Median (IQR)/ \% & \\
\hline \multicolumn{7}{|l|}{ Hematological profile } \\
\hline \multicolumn{7}{|l|}{ Blood group ${ }^{a}$} \\
\hline A & NA & 4 & 26.67 & 5 & 26.32 & \multirow{4}{*}{0.928} \\
\hline$A B$ & NA & 1 & 6.67 & 0 & 0.00 & \\
\hline B & NA & 1 & 6.67 & 2 & 10.53 & \\
\hline $\mathrm{O}$ & NA & 9 & 60.00 & 12 & 63.16 & \\
\hline \multicolumn{7}{|l|}{ Rhesus status $^{a}$} \\
\hline Negative & NA & 0 & 0.00 & 2 & 10.53 & \multirow{2}{*}{0.492} \\
\hline Positive & NA & 15 & 100 & 17 & 89.47 & \\
\hline Het $(\%)^{b}$ & $34.9-44 \cdot 3^{*} / 34 \cdot 7-45.1^{* *}$ & 15 & $34.00(30.80-36.30)$ & 17 & $33.90(30.80-36.40)$ & 0.940 \\
\hline${\text { Leukocyte count }(\mathrm{K} / \mathrm{uL})^{\mathrm{b}}}$ & $4.4-10.6 * / 4.0-11.0^{* *}$ & 15 & $8.80(7.30-11.30)$ & 16 & $9.70(5.70-10.60)$ & 0.621 \\
\hline \multicolumn{7}{|l|}{ Leukopeniac $^{\mathrm{c}}$} \\
\hline Yes $^{a}$ & NA & 2 & 13.33 & 5 & 31.25 & 0.394 \\
\hline Lymphocyte count (K/uL) $^{b}$ & $1.2-3.4^{*} / 0.6-3.4^{\star *}$ & 10 & $16.75(11.00-21.60)$ & 9 & $15.40(11.00-24.60)$ & 0.902 \\
\hline \multicolumn{7}{|l|}{ Lymphopenia $^{d}$} \\
\hline Yes $^{a}$ & NA & 0 & 0.00 & 0 & 0.00 & NA \\
\hline Basophil count (K/uL) ${ }^{b}$ & $0-0.1^{*} / 0-0.2^{* \star}$ & 10 & $0.05(0-0.20)$ & 9 & $0.30(0.30-0.60)$ & 0.035 \\
\hline Neutrophil count $(\mathrm{K} / \mathrm{uL})^{b}$ & $2.2-6.9^{*} 1.7-7.7^{\star *}$ & 10 & $72.70(68.10-76.00)$ & 7 & 72.80 (65.90-77.20) & 1.000 \\
\hline \multicolumn{7}{|l|}{ Neutropenia $^{e}$} \\
\hline Yes $^{a}$ & NA & 0 & 0.00 & 0 & 0.00 & NA \\
\hline Platelet count $(\mathrm{K} / \mathrm{uL})^{\mathrm{b}}$ & $161-369^{*} / 150-450^{* *}$ & 14 & $216.00(157.00-239.00)$ & 16 & $209.50(156.50-239.50)$ & 0.950 \\
\hline \multicolumn{7}{|l|}{ Blood biochemistry profile } \\
\hline $\operatorname{ALT}(U / L)^{b}$ & $5-35^{\star} / 0-50^{\star \star}$ & 5 & $12.00(8.00-20.00)$ & 13 & $12.00(10.00-21.00)$ & 0.519 \\
\hline AST (U/L) ${ }^{b}$ & $0-40 * / 0-40^{* *}$ & 5 & $20.00(20.00-23.00)$ & 13 & 17.00 (15.00-26.00) & 0.236 \\
\hline Glucose $\mathrm{mg} / \mathrm{dL}^{\mathrm{b}}$ & $65-110 * / 70-99^{* *}$ & 7 & 93.00 (69.00-102.00) & 14 & $88.50(74.00-119.00)$ & 1.000 \\
\hline
\end{tabular}




\section{Cureus}

\begin{tabular}{|c|c|c|c|c|c|c|}
\hline Albumin $(g / d L)^{b}$ & $3.8-5.2^{*} / 3.5-5.7^{* \star}$ & ${ }^{4}$ As & 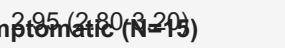 & $18 \mathrm{yr}$ & 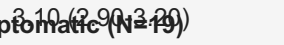 & 0.760 \\
\hline Total bilirubin $(\mathrm{mg} / \mathrm{dL})^{\mathrm{b}}$ & $0.2-1.2^{*} / 0.0-1.0^{* *}$ & $4 \mathbf{n}$ & OAdedf(A.A & $12 n$ & 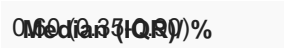 & 0.668 \\
\hline $\operatorname{ALP}(U / L)^{b}$ & $20-120^{*} / 35-104^{\star *}$ & 4 & $198.00(155.00-284.50)$ & 12 & $147.00(95.50-183.50)$ & 0.163 \\
\hline 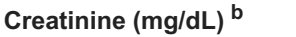 & $0.6-1.4^{\star} / 0.5-1.2^{\star *}$ & 5 & $0.60(0.50-0.80)$ & 12 & $0.53(0.50-0.63)$ & 0.387 \\
\hline $\mathrm{Na}(\mathrm{mEq} / \mathrm{L})^{\mathrm{b}}$ & $135-145^{*} / 133-144^{* *}$ & 6 & $135.50(134.00-137.00)$ & 13 & $136.00(133.00-138.00)$ & 0.894 \\
\hline$K(m E q / L){ }^{b}$ & $3.5-5.0^{*} / 3.5-5.1^{* *}$ & 7 & $4.30(4.00-5.30)$ & 13 & $3.70(3.50-3.90)$ & 0.009 \\
\hline $\mathrm{Ca}(\mathrm{mg} / \mathrm{dL})^{\mathrm{b}}$ & $8.5-10.5^{\star} / 8.6-10.3^{\star \star}$ & 4 & $8.35(8.00-8.65)$ & 12 & $8.20(8.10-8.35)$ & 0.903 \\
\hline BUN (mg/dL) ${ }^{b}$ & $8-20^{*} / 7-25^{\star *}$ & 5 & $5.00(4.00-10.00)$ & 12 & $5.00(4.00-7.00)$ & 0.631 \\
\hline Protein $(g / d L){ }^{b}$ & $6.4-8.3^{*} / 6.4-8.9^{* *}$ & 4 & $5.50(5.05-6.35)$ & 12 & $5.75(5.60-6.00)$ & 0.670 \\
\hline Coagulation profile & & & & & & \\
\hline PT $(s)^{b}$ & $11.9-14.1^{*}$ & 7 & $12.70(11.90-14.60)$ & 7 & $13.10(11.60-13.40)$ & 0.848 \\
\hline $\operatorname{aPTT}(\mathbf{s})^{b}$ & $25.1-36.5^{*}$ & 7 & $31.70(26.20-33.50)$ & 6 & $30.45(30.10-32.00)$ & 0.943 \\
\hline$I_{N R}{ }^{b}$ & $0.9-1.1^{\star \star}$ & 7 & $1.00(0.95-1.17)$ & 7 & $1.01(0.77-1.04)$ & 0.654 \\
\hline Fibrinogen $(\mathrm{mg} / \mathrm{dL})^{b}$ & $178-454^{*} / 163-463^{\star *}$ & 7 & $419.00(364.00-533.00)$ & 6 & $456.00(361.00-533.00)$ & 1.000 \\
\hline Microbiological profile & & & & & & \\
\hline Blood culture ${ }^{a}$ & & & & & & \\
\hline Negative & & 2 & 100.00 & 4 & 80.00 & \\
\hline Positive & & 0 & 0.00 & 1 & 20.00 & \\
\hline Urine culture $^{\mathrm{a}}$ & & & & & & \\
\hline Negative & & 2 & 100 & 3 & 60.00 & \\
\hline Positive & & 0 & 0.00 & 2 & 4.00 & \\
\hline
\end{tabular}

\section{TABLE 2: Laboratory characteristics of pregnant women with COVID-19 by symptom status}

${ }^{*}$ CCHHS normal values, ${ }^{* * A M I T A}$ Saint Mary's normal values

ALP: alkaline phosphate; ALT: alanine aminotransferase; aPTT: activated partial thromboplastin time; AST: aspartate aminotransferase; BUN: blood urea nitrogen; Ca: calcium; Hb: hemoglobin; Hct: Hematocrit; INR: international normalized ratio; K: potassium; Na: sodium; NA: not available; PT: prothrombin time; SD: standard deviation

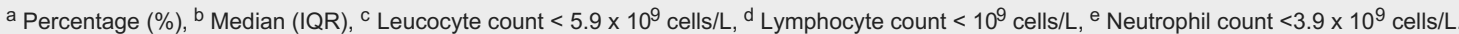

\section{Placental histopathological features}

We found no association of specific histopathological placental features with signs and symptoms of COVID-19 (Table 3). However, we did observe a higher prevalence of fetal vascular malperfusion (FVM), immunological or inflammatory processes, and chorangiosis in symptomatic women compared to asymptomatic women. FVM ( $\mathrm{n}=2$; one avascular villi type and one hemorrhagic endovasculitis type) and chorangiosis $(n=4)$ were observed exclusively in the placentas of symptomatic patients (Figure 4). 


\section{Cureus}

\begin{tabular}{|c|c|c|c|c|c|}
\hline \multirow{2}{*}{ Characteristics } & & Asymptomatic $(\mathrm{N}=15)$ & & Symptomatic $(\mathrm{N}=19)$ & \multirow{2}{*}{$\mathbf{P}$} \\
\hline & $\mathbf{n}$ & Mean (SD)/ \% & $\mathbf{n}$ & Mean (SD)/ \% & \\
\hline \multicolumn{6}{|l|}{ MVM $^{\text {a }}$} \\
\hline No & 3 & 25.00 & 6 & 31.58 & \multirow{2}{*}{1.000} \\
\hline Yes & 9 & 75.00 & 13 & 68.42 & \\
\hline \multicolumn{6}{|l|}{ FVM $^{a}$} \\
\hline No & 12 & 100 & 17 & 89.47 & \multirow{2}{*}{0.510} \\
\hline Yes & 0 & 0.00 & $2^{*}$ & 10.53 & \\
\hline \multicolumn{6}{|l|}{ IM processes ${ }^{\mathrm{a}}$} \\
\hline No & 7 & 58.33 & 7 & 36.84 & \multirow{2}{*}{0.242} \\
\hline Yes & 5 & 41.67 & 12 & 63.16 & \\
\hline \multicolumn{6}{|l|}{ Chorangiosis $^{a}$} \\
\hline No & 12 & 100 & 15 & 78.95 & \multirow{2}{*}{0.139} \\
\hline Yes & 0 & 0.00 & 4 & 21.05 & \\
\hline \multicolumn{6}{|c|}{ Intervillous thrombus ${ }^{a}$} \\
\hline No & 9 & 75.00 & 17 & 89.47 & \multirow{2}{*}{0.350} \\
\hline Yes & 3 & 25.00 & 2 & 10.53 & \\
\hline Placental weight ${ }^{\mathrm{b}}$ & 11 & 489.00 (122.87) & 16 & $506.13(96.44)$ & 0.689 \\
\hline
\end{tabular}

\section{TABLE 3: Placental pathology of pregnant women with COVID-19 by symptom status}

IM: inflammatory or immune process; FVM: fetal vascular malperfusion; MVM: maternal vascular malperfusion; SD: standard deviation.

a Percentage (\%), ${ }^{b}$ Mean (SD), *Avascular villi type $(n=1)$, hemorrhagic endovasculitis type $(n=1)$.

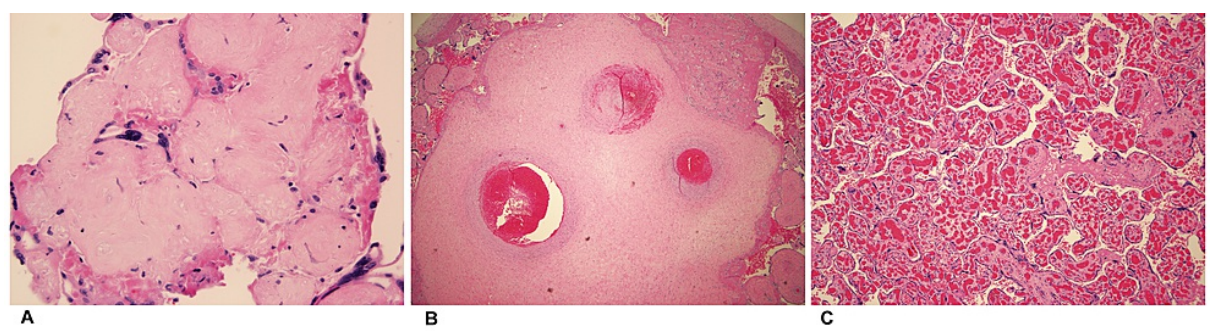

FIGURE 4: Placental pathology unique to symptomatic women with COVID-19 in our study population.

A. Fetal vascular malperfusion (FVM), avascular villi type $(n=1)$; B. FVM, hemorrhagic endovasculitis type $(n=1)$; C. Chorangiosis $(n=4)$.

\section{Logistic regression analysis of significant maternal variables}

Simple logistic regression analysis showed that the odds of severe COVID-19 increased as pre-gestational BMI increased and pre-delivery potassium levels decreased (Table 4). For each unit increase in pregestational BMI, the odds of being symptomatic due to SARS Cov- 2 infection increased by $18 \%$ [odds ratio (OR): 1.18 ; $95 \%$ confidence interval (CI): 1.03 - 1.34)], while a one-unit decrease in serum potassium levels among pregnant women before delivery increased the odds of symptomatic infection by 19.72 (OR: 19.72; 95\% CI: 1.03 - 376.79). 


\section{Cureus}

\begin{tabular}{|c|c|c|}
\hline Variable & Odds ratio (OR) & $95 \% \mathrm{Cl}$ \\
\hline Pre-pregnancy BMI (kg/m2) & 1.18 & $(1.03-1.34)$ \\
\hline Potassium (mEq/L)* & 19.72 & $(1.03-376.79)$ \\
\hline
\end{tabular}

\section{TABLE 4: Simple logistic regression model of predictors of symptomatic COVID-19 in pregnant}

women.

*In the calculation of the odds ratio for potassium, negative one unit (i.e., unit $=-1$ ) was used to calculate the increase in the odds of symptomatic infection per one unit decrease in potassium level.

Cl: Confidence interval.

\section{Fetal and neonatal outcomes}

Among 34 deliveries, there were two twin deliveries culminating in a total of 36 infants in our study population (Table 5). Mean birth weight was $3332.50 \mathrm{~g}$ ( $\mathrm{SD}=778.37$ ) with a median GA of 39.10 weeks (IQR: 38.00-40.00). The average birth weight of babies born to symptomatic women was significantly higher than that of asymptomatic women (mean: $3454.00 \mathrm{~g}$ [SD: 521.11] vs 2885.80g [SD: 941.09], $\mathrm{P}=0.048$ ). We observed three premature deliveries (GA<37 weeks), with one resulting in twin fetal demise. In contrast to the mothers, the majority of newborns were asymptomatic, with tachypnea most likely secondary to transient tachypnea observed as the most common symptom in five symptomatic newborns. Among five newborns with tachypnea, four were born to symptomatic mothers. However, none of the newborns tested positive for COVID-19.

\begin{tabular}{|c|c|c|c|c|c|}
\hline \multirow{2}{*}{ Characteristics } & & Asymptomatic (N=15) & & Symptomatic $(\mathrm{N}=19)$ & \multirow{2}{*}{$\mathbf{P}$} \\
\hline & $\mathbf{n}$ & $\begin{array}{l}\text { Mean (SD)/ Median }(\text { (QR)/ } \\
\%\end{array}$ & $\mathbf{n}$ & $\begin{array}{l}\text { Mean (SD)/ Median (IQR)/ } \\
\%\end{array}$ & \\
\hline Weight of newborns, $g^{a}$ & 15 & $2885.80(941.09)$ & 19 & $3454.00(521.11)$ & 0.048 \\
\hline Premature birth $\left(<37\right.$ weeks) ${ }^{b}$ & 3 & 21.43 & 0 & 0.00 & 0.067 \\
\hline Stillbirth*b & 2 & 13.33 & 0 & 0.00 & \\
\hline \multicolumn{6}{|l|}{ Sex ${ }^{b}$} \\
\hline Male & 9 & 60.00 & 9 & 47.37 & \multirow{2}{*}{0.464} \\
\hline Female & 6 & 40.00 & 10 & 52.63 & \\
\hline Apgar scores at 1 minute ${ }^{c}$ & 15 & $9.00(9.00-9.00)$ & 19 & $9.00(8.00-9.00)$ & 0.790 \\
\hline Apgar scores at 5 minutes ${ }^{c}$ & 15 & $9.00(9.00-9.00)$ & 19 & $9.00(9.00-9.00)$ & 0.724 \\
\hline \multicolumn{6}{|l|}{ RT PCR for SARS-Cov2 ${ }^{b}$} \\
\hline Positive & 0 & 0.00 & 0 & 0.00 & \multirow{3}{*}{ NA } \\
\hline Negative & 15 & 100.00 & 13 & 68.42 & \\
\hline Not done & 1 & 0.00 & 6 & 31.58 & \\
\hline Initial temperature & 15 & $36.80(36.50-37.10)$ & 18 & $36.70(36.50-37.00)$ & 0.971 \\
\hline \multicolumn{6}{|l|}{ Symptoms ${ }^{b}$} \\
\hline Apnea & 1 & 7.14 & 0 & 0.00 & 0.424 \\
\hline Increased work of breathing & 2 & 14.29 & 3 & 15.79 & 1.000 \\
\hline Desaturations & 2 & 14.29 & 4 & 21.05 & 0.618 \\
\hline Hypoglycemia & 0 & 0.00 & 1 & 5.26 & 1.000 \\
\hline Poor feeding & 0 & 0.00 & 2 & 10.53 & 0.496 \\
\hline
\end{tabular}




\section{Cureus}

\begin{tabular}{|c|c|c|c|c|c|}
\hline Fever & 1 & Asymptomatic $(\mathrm{N}=15)$ & 0 & S.Symptomatic ( $\mathrm{N}=19)$ & 0.424 \\
\hline $\begin{array}{l}\text { Characteristics } \\
\text { Diagnosis }\end{array}$ & $\mathbf{n}$ & $\begin{array}{l}\text { Mean (SD)/ Median (IQR)/ } \\
\%\end{array}$ & $\mathbf{n}$ & $\begin{array}{l}\text { Mean (SD)/ Median (IQR)/ } \\
\%\end{array}$ & $\mathbf{P}$ \\
\hline Normal & 9 & 60.00 & 9 & 50.00 & 0.566 \\
\hline RDS & 2 & 13.33 & 1 & 5.26 & 0.571 \\
\hline Rule out sepsis & 3 & 20.00 & 0 & 0.00 & 0.076 \\
\hline TTN & 0 & 0.00 & 2 & 10.53 & 0.492 \\
\hline Apnea & 1 & 6.67 & 0 & 0.00 & 0.441 \\
\hline Pneumomediastinum & 0 & 0.00 & 1 & 5.26 & 1.000 \\
\hline Poor feeding/GERD & 0 & 0.00 & 1 & 5.26 & 1.000 \\
\hline Hypoglycemia & 0 & 0.00 & 1 & 5.26 & 1.000 \\
\hline Birth Injury & 0 & 0.00 & 1 & 5.26 & 1.000 \\
\hline \multicolumn{6}{|l|}{ Disposition $^{b}$} \\
\hline SCN/NICU visit and discharged & 6 & 40.00 & 9 & 50.00 & \multirow[b]{2}{*}{0.566} \\
\hline $\begin{array}{l}\text { Nursery/Postpartum floor visit and } \\
\text { discharged }\end{array}$ & 9 & 60.00 & 9 & 50.00 & \\
\hline
\end{tabular}

\section{TABLE 5: Clinical and demographic characteristics of infants born to women with COVID-19, by}

maternal symptom status

*Includes one pair of twins

NA: not available; RDS: respiratory distress syndrome; RT-PCR: reverse transcriptase-polymerase chain reaction; TTN: transient tachypnea of newborn; SCN/NICU: Special Care Nursery/ Neonatal Intensive Care Unit; GERD: gastroesophageal reflux disease.

${ }^{a}$ Mean (SD), ${ }^{\text {b }}$ Median (IQR), ${ }^{\mathrm{c}}$ Percentage (\%)

\section{Laboratory characteristics of newborns}

We did not detect any difference in laboratory characteristics in infants of symptomatic women compared with those of asymptomatic women (Table 6). We also measured total $\operatorname{IgM}(n=6)$ and total IgG $(n=7)$ antibodies in a limited number of infants (Table 6). Our data suggest higher levels of IgM antibodies in one infant (17.00) born to a symptomatic mother compared with four infants born to asymptomatic mothers (median: 10.00, IQR: 9.00-11.00), but the low sample size makes it impossible to make any meaningful clinical interpretation. 


\section{Cureus}

\begin{tabular}{|c|c|c|c|c|c|c|}
\hline \multirow{2}{*}{ Characteristics } & \multirow{2}{*}{ Normal Range } & \multicolumn{2}{|c|}{ Asymptomatic (N=15) } & \multicolumn{2}{|c|}{ Symptomatic (N=19) } & \multirow{2}{*}{$\mathbf{P}$} \\
\hline & & $\mathbf{n}$ & Median (IQR)/ \% & $\mathbf{n}$ & Median (IQR)/ \% & \\
\hline \multicolumn{7}{|l|}{ Hematological profile } \\
\hline Hct (\%) ${ }^{a}$ & $42-60.0 * / 42.0-54.0^{* *}$ & 6 & $46.40(42.35-53.80)$ & 14 & $50.90(47.30-52.70)$ & 0.322 \\
\hline Leucocyte count (K/uL) a & $9.1-34.0 * / 8.0-15.4^{* *}$ & 8 & $13.00(9.80-17.95)$ & 14 & $13.85(11.30-17.30)$ & 0.657 \\
\hline \multicolumn{7}{|l|}{ Leukopenia $^{c}$} \\
\hline Yes $^{b}$ & NA & 2 & 28.57 & 5 & 35.71 & 1.000 \\
\hline${\text { Lymphocyte count }(\%)^{a}}^{a}$ & $11.0-30.9^{*} / 33.7-67.6^{* *}$ & 7 & $26.00(19.00-32.00)$ & 14 & $22.00(14.00-30.00)$ & 0.501 \\
\hline \multicolumn{7}{|l|}{ Lymphopenia $^{d}$} \\
\hline Yes $^{b}$ & NA & 0 & 0.00 & 0 & 0.00 & NA \\
\hline Basophil count (\%) ${ }^{a}$ & $0.0-0.3^{*} / 0.1-0.8^{\star *}$ & 5 & $2.00(0.00-5.00)$ & 7 & $0.00(0.00-1.00)$ & 0.487 \\
\hline Monocyte count (\%) ${ }^{a}$ & $0.0-10.0 * / 6.7-19.9^{* *}$ & 3 & $9.00(8.00-12.00)$ & 8 & $13.20(8.50-15.90)$ & 0.357 \\
\hline Neutrophil count (\%) ${ }^{a}$ & $65.9-69.1^{*} / 20.2-46.2^{* *}$ & 7 & $50.00(45.00-58.00)$ & 14 & $60.50(52.00-73.00)$ & 0.192 \\
\hline \multicolumn{7}{|l|}{ Neutropenia $^{\mathrm{e}}$} \\
\hline Yes $^{b}$ & NA & 0 & 0.00 & 0 & 0.00 & NA \\
\hline Platelet count (K/uL) ${ }^{a}$ & $200-400 * / 145-262^{* *}$ & 8 & $192.00(179.00-221.50)$ & 14 & $245.50(150.00-290.00)$ & 0.232 \\
\hline Eosinophils count $(\%)^{a}$ & $0.3-5.2^{* *}$ & 3 & $3.00(4.00-4.00)$ & 6 & $1.55(3.00-3.00)$ & 0.691 \\
\hline \multicolumn{7}{|l|}{ Blood biochemistry profile } \\
\hline $\operatorname{CRP}(\mathrm{mg} / \mathrm{dL})^{\mathrm{a}}$ & $<1.0^{\star \star}$ & 3 & $0.10(0.07-2.09)$ & 8 & $0.09(0.04-0.21)$ & 0.409 \\
\hline $\operatorname{ALT}(U / L)^{a}$ & $5-35^{*} / 0.0-40.0^{* *}$ & 3 & $10.00(9.00-17.00)$ & 6 & $13.50(11.00-19.00)$ & 0.519 \\
\hline AST (U/L) ${ }^{a}$ & $0-40 * / 0-32^{* *}$ & 2 & $42.50(25.00-60.00)$ & 5 & $49.00(37.00-57.00)$ & 0.847 \\
\hline Creatinine $(\mathrm{mg} / \mathrm{dL})^{\mathrm{a}}$ & $0.6-1.4^{*} / 0.5-1.2^{* *}$ & 3 & $0.70(0.70-0.80)$ & 6 & $0.86(0.60-1.00)$ & 0.693 \\
\hline BUN $(m g / d L){ }^{a}$ & $8-20^{*} / 7-25^{\star *}$ & 3 & $9.00(7.00-28.00)$ & 5 & $8.00(6.00-8.00)$ & 0.368 \\
\hline \multicolumn{7}{|l|}{ Blood culture $^{b}$} \\
\hline Negative & & 7 & 100 & 9 & 100 & \multirow{2}{*}{ NA } \\
\hline Positive & & 0 & 0.00 & 0 & 0.00 & \\
\hline \multicolumn{7}{|l|}{ Immunological profile } \\
\hline $\lg M(m g / d L)^{a}$ & $3-13^{*} / 14-142$ ** & 5 & $10.00(9.00-11.00)$ & 1 & 17.00 (17.00-17.00) & 0.373 \\
\hline $\lg G(\mathrm{mg} / \mathrm{dL})^{a}$ & $74-1421^{*}$ & 5 & $724.00(706.00-868.00)$ & 2 & $560.00(20.00-1100.00)$ & 0.847 \\
\hline
\end{tabular}

\section{TABLE 6: Laboratory characteristics of infants born to women with COVID-19, by maternal}

symptom status

* CCHHS normal values, **AMITA Saint Marys normal values; ALT: alanine aminotransferase, AST: aspartate aminotransferase, BUN: blood urea nitrogen, CRP: C-reactive protein, Hct: Hematocrit, Ig: immunoglobulin, NA: not available.

${ }^{a}$ Median (IQR), ${ }^{b}$ Percentage (\%), ${ }^{\mathrm{C}}$ Leucocyte count $<9.0 \times 10^{9}$ cells $/ \mathrm{L}$ for preterm infants and $13.0 \times 10^{9}$ cells $/ \mathrm{L}$ for term infants, ${ }^{\mathrm{d}}$ Lymphocyte count $<2.0 \times 10^{9}$ cells $/$ L, ${ }^{\text {e }}$ Neutrophil count $<6.0 \times 10^{9}$ cells $/ \mathrm{L}$. 


\section{Discussion}

To the best of our knowledge, this is the largest cohort of mother-baby dyads (SARS-Cov-2-positive women and their newborn infants) to undergo detailed clinical and biochemical investigation in the state of Illinois. In this study, high BMI and low potassium levels were associated with symptomatic COVID-19 in mothers.

About half of pregnant women in our study developed symptoms of COVID-19, which falls in the middle range of similar studies. A recent study of 70 pregnant women with SARS-CoV-2 in New York City reported that only $21 \%$ presented with symptoms [22], while an earlier study from Wuhan, China showed $95 \%$ of pregnant women with the virus exhibited symptoms [23].

We found that women with a higher pre-gestational BMI were more likely to be symptomatic, consistent with several reports showing an association between pre-gestational BMI with severe maternal outcomes among women with COVID-19 [12,14]. An Italian study observed significantly higher pre-gestational BMI in seven of 14 women showing severe symptoms [12], and a case series from Washington State found that the majority of pregnant women with severe infection were overweight or obese [14].

Our finding of significantly lower potassium levels in symptomatic women is consistent with a report of lower potassium levels (<3.5 mEq/L) in 119 patients (41\%) of 290 with COVID-19 in the general population [24]. In that study, hypokalemic patients were more likely to stay longer in hospitals, with a higher rate of respiratory symptoms. SARS-CoV-2 enters cells by binding to angiotensin-converting enzyme 2 (ACE2), leading to ACE2 depletion in affected cells [25]. ACE2 depletion promotes vasoconstriction, increasing the reabsorption of water and sodium [26]. These changes could lead to increased potassium excretion in symptomatic patients who may have higher viral loads [27]. Also, the contribution of respiratory alkalosis or diarrhea to low potassium levels among pregnant women with COVID-19 cannot be ruled out. Since low potassium levels could lead to life-threatening conditions, including cardiac events, our findings suggest the need to monitor serum potassium and improve care for pregnant women with COVID-19 by ensuring adequate potassium supplementation.

Hispanic and non-Hispanic Black populations comprised $44 \%$ and $38 \%$ of the pregnant women in our study population, respectively. A recent study of 1,567 Hispanic pregnant and postpartum women identified obesity as a major risk factor for moderate and severe COVID-19 [28], consistent with our finding that BMI was associated with symptomatic infection. We did not observe an association between Hispanic race/ethnicity and symptomatic COVID-19, but ours was a small study, and a larger study encompassing a broader geographic area may have yielded different results.

Our results also showed significantly higher basophil cell count in symptomatic patients compared with asymptomatic patients. A recent study demonstrated an association of elevated basophil levels with the IgG antibodies against SARS-CoV-2 produced by B cells during the disease's recovery phase, suggesting the possibility of heightened humoral response in symptomatic pregnant women [29].

We did not find a statistically significant association of specific placental pathologies with COVID-19 symptom status among the women in our study, and this result is consistent with a recent report by Hecht et al [30]. However, we did observe a spectrum of histopathologies (chorangiosis and FVM ) exclusively among symptomatic women, which were similar to inflammatory changes observed in placentas from a case series of fetal demise associated with maternal SARS-Cov-2 infection [16].

\section{Limitations}

Our study is limited by the small sample size and the retrospective study design. The retrospective data collection contributed to missing values and an increased likelihood of information bias. The sample size was too small to perform a robust multivariable analysis, so we were unable to assess potential predictive variables or evaluate confounding. However, our posthoc power analysis showed that our sample size of 34 women achieved a power of $92 \%$, which is higher than the $80 \%$ standard generally seen in the literature. Finally, because the participating hospitals draw from largely an urban population of Chicago, our findings may not be generalizable to communities in other areas of the United States.

Even so, our findings have important implications for the clinical management of pregnant women with COVID-19. Our study suggests that pregnant women who test positive for SARS-CoV-2 should be closely monitored for a rapid progression of symptoms, especially women with a high pre-gestational BMI and/or low potassium levels. However, it remains to be seen whether low potassium levels are a cause or outcome of symptoms observed in pregnant women with COVID-19.

\section{Conclusions}

The implications of SARS-CoV-2 infection in pregnant women are still not fully understood. However, our study observed that some pregnant women may be especially vulnerable to symptomatic infection. Our case series found that women with a high pregestational BMI had a higher risk of developing symptomatic COVID-19. The lower potassium levels we observed in symptomatic women could be a function of disease 
severity, or alternatively, could be a causal factor for the development of symptoms. We are unable to discern causality because of the retrospective nature of the study. Nevertheless, our study validates previous reports of an association of potassium levels with disease severity. We thereby strongly recommend closely following potassium levels to monitor disease severity in pregnant women with COVID-19 infection. Potassium levels may also be useful as a biomarker of symptom development. Finally, although it is encouraging that none of the newborns in our study tested positive for the virus, further research on the risks of vertical transmission for infants of women with COVID-19 during pregnancy is needed.

\section{Additional Information \\ Disclosures}

Human subjects: Consent was obtained or waived by all participants in this study. John H. Stroger, Jr. Hospital of Cook County issued approval 20-098. The study's protocol was approved by the Institutional Review Board associated with John H. Stroger, Jr. Hospital of Cook County, and informed consent was waived due to the retrospective study design. Animal subjects: All authors have confirmed that this study did not involve animal subjects or tissue. Conflicts of interest: In compliance with the ICMJE uniform disclosure form, all authors declare the following: Payment/services info: All authors have declared that no financial support was received from any organization for the submitted work. Financial relationships: All authors have declared that they have no financial relationships at present or within the previous three years with any organizations that might have an interest in the submitted work. Other relationships: All authors have declared that there are no other relationships or activities that could appear to have influenced the submitted work.

\section{Acknowledgements}

The authors would like to thank Mohammed Siddiqui, BS, for his assistance in editing the slides for the presentation of this study at the meeting of the Pediatric Academic Societies (PAS), and Helen Atkinson, MD for her assistance with manuscript preparation.

\section{References}

1. Chen H, Guo J, Wang C, et al.: Clinical characteristics and intrauterine vertical transmission potential of COVID-19 infection in nine pregnant women: a retrospective review of medical records. Lancet. 2020, 395:809-15. 10.1016/S0140-6736(20)30360-3

2. Dell'Era V, Farri F, Garzaro G, Gatto M, Aluffi Valletti P, Garzaro M: Smell and taste disorders during COVID-19 outbreak: Cross-sectional study on 355 patients. Head Neck. 2020, 42:1591-6. 10.1002/hed.26288

3. Kumar A, Arora A, Sharma P, et al.: Gastrointestinal and hepatic manifestations of Corona Virus Disease-19 and their relationship to severe clinical course: A systematic review and meta-analysis. Indian J Gastroenterol. 2020, 39:268-84. 10.1007/s12664-020-01058-3

4. Li J, Huang DQ, Zou B, et al.: Epidemiology of COVID-19: a systematic review and meta-analysis of clinical characteristics, risk factors, and outcomes. J Med Virol. 2021, 93:1449-58. 10.1002/jmv.26424

5. Chin-Hong P, Alexander KM, Haynes N, Albert MA: Pulling at the heart: COVID-19, race/ethnicity and ongoing disparities. Nat Rev Cardiol. 2020, 17:533-5. 10.1038/s41569-020-0416-6

6. Dai CL, Kornilov SA, Roper RT, et al.: Characteristics and factors associated with Coronavirus Disease 2019 infection, hospitalization, and mortality across race and ethnicity. Clin Infect Dis. 2021, 73:2193-204. 10.1093/cid/ciab154

7. Zhu H, Wang L, Fang C, et al.: Clinical analysis of 10 neonates born to mothers with 2019-nCoV pneumonia . Transl Pediatr. 2020, 9:51-60. 10.21037/tp.2020.02.06

8. Walker KF, O'Donoghue K, Grace N, Dorling J, Comeau JL, Li W, Thornton JG: Maternal transmission of SARS-COV-2 to the neonate, and possible routes for such transmission: a systematic review and critical analysis. BJOG. 2020, 127:1324-36. 10.1111/1471-0528.16362

9. Villar J, Ariff S, Gunier RB, et al.: Maternal and neonatal morbidity and mortality among pregnant women with and without COVID-19 infection: the INTERCOVID multinational cohort study. JAMA Pediatr. 2021, 175:817-26. 10.1001/jamapediatrics.2021.1050

10. London V, McLaren R Jr, Atallah F, et al.: The relationship between status at presentation and outcomes among pregnant women with COVID-19. Am J Perinatol. 2020, 37:991-4. 10.1055/s-0040-1712164

11. Hantoushzadeh S, Shamshirsaz AA, Aleyasin A, et al.: Maternal death due to COVID-19. Am J Obstet Gynecol. 2020, 223:109.e1-109.e16. 10.1016/j.ajog.2020.04.030

12. Savasi VM, Parisi F, Patanè L, et al.: Clinical findings and disease severity in hospitalized pregnant women with Coronavirus Disease 2019 (COVID-19). Obstet Gynecol. 2020, 136:252-8. 10.1097/AOG.0000000000003979

13. Sentilhes L, De Marcillac F, Jouffrieau C, et al.: Coronavirus disease 2019 in pregnancy was associated with maternal morbidity and preterm birth. Am J Obstet Gynecol. 2020, 223:914.e1-914.e15. 10.1016/j.ajog.2020.06.022

14. Lokken EM, Walker CL, Delaney S, et al.: Clinical characteristics of 46 pregnant women with a severe acute respiratory syndrome coronavirus 2 infection in Washington State. Am J Obstet Gynecol. 2020, 223:911.e1911.e14. 10.1016/j.ajog.2020.05.031

15. Vivanti AJ, Mattern J, Vauloup-Fellous C, et al.: Retrospective description of pregnant women infected with severe acute respiratory syndrome Coronavirus 2, France. Emerg Infect Dis. 2020, 26:2069-76. 10.3201/eid2609.202144

16. Richtmann R, Torloni MR, Oyamada Otani AR, et al.: Fetal deaths in pregnancies with SARS-CoV-2 infection in Brazil: A case series. Case Rep Womens Health. 2020, 27:e00243. 10.1016/j.crwh.2020.e00243 
17. World Health Organization. Laboratory testing for coronavirus disease 2019 (COVID-19) in suspected human cases: interim guidance, 2 March 2020. (2020). Accessed: June 28, 2021 : https://apps.who.int/iris/handle/10665/331329.

18. Abbassi-Ghanavati M, Greer LG, Cunningham FG: Pregnancy and laboratory studies: a reference table for clinicians. Obstet Gynecol. 2009, 114:1326-31. 10.1097/AOG.0b013e3181c2bde8

19. Khong TY, Mooney EE, Ariel I, et al.: Sampling and definitions of placental lesions: Amsterdam Placental Workshop Group Consensus Statement. Arch Pathol Lab Med. 2016, 140:698-713. 10.5858/arpa.2015-0225CC

20. Rennie JM, Roberton NRC: Textbook of Neonatology. Edinburgh: Churchill Livingstone . Rennie JM, Roberton NRC (ed): Churchill Livingstone, Edinburgh; 1999.

21. Evaluation and management considerations for neonates at risk for COVID-19 . (2020). Accessed: June 28, 2021: https://stacks.cdc.gov/view/cdc/88194.

22. Prabhu M, Cagino K, Matthews KC, et al.: Pregnancy and postpartum outcomes in a universally tested population for SARS-CoV-2 in New York City: a prospective cohort study. BJOG. 2020, 127:1548-56. 10.1111/1471-0528.16403

23. Chen L, Li Q, Zheng D, et al.: Clinical characteristics of pregnant women with Covid-19 in Wuhan, China . N Engl J Med. 2020, 382:e100. 10.1056/NEJMc2009226

24. Alfano G, Ferrari A, Fontana F, et al.: Hypokalemia in patients with COVID-19. Clin Exp Nephrol. 2021, 25:401-9. 10.1007/s10157-020-01996-4

25. Chen D, Li X, Song Q, et al.: Assessment of hypokalemia and clinical characteristics in patients with Coronavirus Disease 2019 in Wenzhou, China. JAMA Netw Open. 2020, 3:e2011122.

10.1001/jamanetworkopen.2020.11122

26. Guo J, Huang Z, Lin L, Lv J: Coronavirus disease 2019 (COVID-19) and cardiovascular disease: a viewpoint on the potential influence of angiotensin-converting enzyme inhibitors/angiotensin receptor blockers on onset and severity of severe acute respiratory syndrome coronavirus 2 infection. J Am Heart Assoc. 2020, 9:e016219. 10.1161/JAHA.120.016219

27. Weir MR, Rolfe M: Potassium homeostasis and renin-angiotensin-aldosterone system inhibitors . Clin J Am Soc Nephrol. 2010, 5:531-48. 10.2215/CJN.07821109

28. Grechukhina O, Greenberg V, Lundsberg LS, et al.: Coronavirus disease 2019 pregnancy outcomes in a racially and ethnically diverse population. Am J Obstet Gynecol MFM. 2020, 2:100246. 10.1016/j.ajogmf.2020.100246

29. Rodriguez L, Pekkarinen PT, Lakshmikanth T, et al.: Systems-level immunomonitoring from acute to recovery phase of severe COVID-19. Cell Rep Med. 2020, 1:100078. 10.1016/j.xcrm.2020.100078

30. Hecht JL, Quade B, Deshpande V, et al.: SARS-CoV-2 can infect the placenta and is not associated with specific placental histopathology: a series of 19 placentas from COVID-19-positive mothers. Mod Pathol. 2020, 33:2092-103. 10.1038/s41379-020-0639-4 